\title{
Quintessence in low-energy effective theory
}

\author{
Tae Hoon Lee ${ }^{1,2, \star}$ \\ ${ }^{1}$ Department of Physics, Soongsil University, Seoul 06978, Korea \\ ${ }^{2}$ Research Institute for Origin of Matter and Evolution of Galaxies, Soongsil University, Seoul 06978, Korea
}

\begin{abstract}
Considering a theory of Brans-Dicke gravity with general couplings of a heavy field, we derive the low-energy effective theory action in the universe of temperature much lower than the heavy field mass. Gravitational equations and the Brans-Dicke scalar field equation including an effective potential of the scalar field are obtained, which is induced through virtual interactions of the heavy field in the late-time universe. We find a deSitter cosmological solution stemming from the inverse power law effective potential of the scalar field and discuss the possibility that the late time acceleration of our universe can be described by means of the solution.
\end{abstract}

Recent cosmological observations including supernova data [1] suggest that our universe is made up of about $68 \%$ dark energy, about $27 \%$ dark matter, and about $5 \%$ ordinary matter, and they cause an explosion of interest in the origin of dark energy [1] and dark matter [2]. The current cosmic acceleration due to the dark energy and the primordial inflation can be described by using the de Sitter solutions to the gravitational field equations. Various approaches have been used to understand the dark energy, such as the cosmological constant, quintessence [3], and the phantom [4] in general relativity. Other approaches are based on assorted types of modifications to general relativity, such as $f(R)$ gravity and scalar-tensor theories of gravity. We study the late-time acceleration of the Universe, in the Brans-Dicke (BD) theory of gravity coupled to a heavy field.

From the BD gravity theory, we derive the low-energy effective theory [5] action in the universe of temperature much lower than the heavy field mass and obtain an inverse power law effective potential of the BD scalar field, which is stimulated through virtual interactions of the heavy field. With the effective potential we discuss the possibility that the cosmological constant problem can be addressed. Before doing these things, we present the method by which the low-energy effective theory can be obtained from a high energy theory, as follows:

The (effective action or) generating functional (GF) for connected, one-particle-irreducible (1PI) with respect to $\phi$ - and $h$-lines Green functions is given by the Legendre transform of a GF for connected Green functions, $W(j, J)=-i \ln Z(j, J)$ with GF for (general) Green functions $Z(j, J)$,

$$
\Gamma(\phi, h)=W(j, J)-\int d^{4} x j \phi-\int d^{4} x J h
$$

with $\frac{\delta W(j, J)}{\delta j}=\phi, \frac{\delta W(j, J)}{\delta J}=h$. Eq. (1) can be rewritten as

$$
\Gamma(\phi, h)=\Gamma(\phi, J)-\int d^{4} x J h,
$$

\footnotetext{
^e-mail: thlee@ssu.ac.kr
} 
where the GF for connected, 1PI with respect to $\phi$-lines Green functions is given by the partial Legendre transform

$$
\Gamma(\phi, J)=W(j, J)-\int d^{4} x j \phi .
$$

with $\frac{\delta W(j, J))}{\delta j}=\phi$. From (2) we also have the relation

$$
\Gamma(\phi, J)=\Gamma(\phi, h)+\int d^{4} x J h
$$

with

$$
\frac{\delta \Gamma(\phi, h)}{\delta h}=-J
$$

which gives us $h=h(\phi, J)$.

When the freedoms associated with matter fields, $h\left(x^{\mu}\right)$, heavier $\left(m_{h}>T\right)$ than the temperature of the Universe are hidden from direct observation (i.e., $J=0$ ), the effective theory is described by the low-energy effective action $S_{e f f}(\phi)$ of light fields, $\phi\left(x^{\mu}\right)$, only. In the tree-level approximation, its action is given by

$$
S_{e f f}(\phi) \equiv \Gamma^{(0)}(\phi, h(\phi))
$$

where $h(\phi) \equiv h(\phi, J=0)$ is derived from the equation

$$
\frac{\delta \Gamma^{(0)}(\phi, h)}{\delta h}=0
$$

with the effective action $\Gamma(\phi, h)$ defined in terms of both $\phi$ and $h$ fields in a high-energy theory, as in Eq. (1).

The BD theory of gravity coupled to a heavy field $h\left(x^{\mu}\right)$ can be described by the action, $S=$ $\int d^{4} x \sqrt{-g}\left[\varphi R-\frac{\omega_{b d}}{\varphi} g^{\alpha \beta} \partial_{\alpha} \varphi \partial_{\beta} \varphi-V(\varphi)\right]+S_{(h m)}$, which is rewritten as

$$
S=\int d^{4} x \sqrt{-g}\left[\phi^{2} R-\omega g^{\alpha \beta} \partial_{\alpha} \phi \partial_{\beta} \phi-V(\phi)\right]+S_{(h m)},
$$

with substitution $\varphi=\phi^{2}$ and $\omega_{b d}=\frac{1}{4} \omega$. The action for the heavy field is assumed by

$$
S_{(h m)}=\int d^{4} x \sqrt{-g}\left[-\frac{1}{2} g^{\alpha \beta} \partial_{\alpha} h \partial_{\beta} h-\bar{V}(h)-V_{\text {int }}(\phi, h)\right],
$$

where $\bar{V}$ is the potential of the heavy field and the non-renormalizable interaction potential

$$
V_{\text {int }}(\phi, h)=\frac{u}{M_{d}^{k+l-4}} \phi^{k} h^{l}
$$

with $k+l>4$.

By applying the formula (7) to Eqs. (9) and (10) and by choosing $\bar{V}(h)=\frac{1}{2} m_{h}^{2} h^{2}$, we get

$$
h(\phi) \simeq\left(\frac{-u l \phi^{k}}{m_{h}^{2} M_{d}^{(k+l-4)}}\right)^{\frac{1}{2-l}}\left(1+\frac{1}{2-l} \frac{g^{\alpha \beta} \nabla_{\alpha} \partial_{\beta}}{m_{h}^{2}}\right) \simeq\left(\frac{-u l \phi^{k}}{m_{h}^{2} M_{d}^{(k+l-4)}}\right)^{\frac{1}{2-l}},
$$

in the low-energy limit, $\left|\partial_{\beta} h\right|<<m_{h} h$. Substituting Eq. (11) into Eqs. (6) and (8), we have

$$
S_{e f f}(\phi) \simeq \int d^{4} x \sqrt{-g}\left[\phi^{2} R-\omega(\phi) g^{\alpha \beta} \partial_{\alpha} \phi \partial_{\beta} \phi-V(\phi)-\frac{1}{2} g^{\alpha \beta} \partial_{\alpha} h(\phi) \partial_{\beta} h(\phi)-\mathcal{V}^{e f f}(\phi)\right],
$$


where the effective potential

$$
\mathcal{V}^{e f f}(\phi)=d \phi^{\frac{-2 k}{1-2}}
$$

with $d=\left(1 / 2 l^{\frac{2}{2-l}}-l^{\frac{l}{2-l}}\right)(-u)^{\frac{2}{2-l}} m_{h}^{\frac{2 l}{l-2}}\left(M_{d}^{(k+l-4)}\right)^{\frac{2}{l-2}}(u<0, k+l>4$, and $l>2)$, which gives us an inverse power law potential for a quintessence model. The equation of state is given by

$$
\omega_{t o t} \equiv \frac{p}{\rho}=\frac{\omega \dot{\phi}^{2}+4\left(\dot{\phi}^{2}+\phi \ddot{\phi}+2 H \phi \dot{\phi}\right)-V+c \phi^{\frac{2 k+2 l-4}{2-l}} \dot{\phi}^{2}-d \phi^{\frac{-2 k}{1-2}}}{\omega \dot{\phi}^{2}-12 H \phi \dot{\phi}+V+c \phi^{\frac{2 k+2 l-4}{2-l}} \dot{\phi}^{2}+d \phi^{\frac{2 k}{l-2}}}
$$

with $c=\frac{1}{2} \frac{k^{2}}{(2-l)^{2}}\left(\frac{-u l}{m_{h}^{2} M_{d m}^{(k+1-4)}}\right) \frac{2}{2-l}$. Note that $\omega_{t o t}>-1$.

In the flat Friedamnn-Robertson-Waker spacetime, we can derive the scalar field equation and the Friedmann equation from Eq. (12).

$$
\begin{aligned}
& \left(2 \omega+h^{\prime 2}\right) \ddot{\phi} \\
& =-\left(6 \omega H+3 H h^{\prime 2}+h^{\prime} h^{\prime \prime} \dot{\phi}\right) \dot{\phi}-2\left\{V_{\phi^{2}}-6\left(\dot{H}+2 H^{2}\right)+\mathcal{V}^{e f f}{ }_{\phi^{2}}\right\} \phi
\end{aligned}
$$

with $h^{\prime} \equiv \frac{\partial h}{\partial \phi}, V_{\phi^{2}} \equiv \frac{\partial V}{\partial \phi^{2}}, \ldots$ and

$$
\begin{aligned}
& -4 \phi^{2} \dot{H}(=\rho+p) \\
& =2(\omega+2) \dot{\phi}^{2}+4 \phi \ddot{\phi}+\dot{h}^{2}(\phi)-4 H \phi \dot{\phi},
\end{aligned}
$$

subject to the constraint

$$
6 \phi^{2} H^{2}=\omega \dot{\phi}^{2}-12 H \phi \dot{\phi}+V+\frac{1}{2} \dot{h}^{2}(\phi)+\mathcal{V}^{e f f}(\phi),
$$

from which we get a solution for de Sitter universe with $\dot{H}=0$ and $\ddot{\phi}=0$, which has following requirements (with $H=$ const. and $\dot{\phi}=$ const.):

$$
\begin{aligned}
6 \phi^{2} H^{2}=\omega_{e} \dot{\phi}^{2}-12 \phi \dot{\phi} H+V(\phi) & +\mathcal{V}^{e f f}(\phi), \\
& \left(\omega_{e}+2\right) \dot{\phi}^{2}-2 H \phi \dot{\phi}=0,
\end{aligned}
$$

and

$$
\left(6 \omega_{e} H+h^{\prime} h^{\prime \prime} \dot{\phi}\right) \dot{\phi}=\left\{2 V, \phi_{\phi^{2}}-24 H^{2}+2 \mathcal{V}^{e f f},{ }_{\phi^{2}}\right\} \phi
$$

with $\omega_{e} \equiv \omega\left(1+\frac{h^{\prime 2}}{2 \omega}\right)$. Linear stability analyses near the above solution are given in [6].

When $\dot{\phi}^{2}<<1$ and $V(\phi)<<1$ are assumed, Eq. (16) yields $\Lambda \phi^{2} \sim \mathcal{V}^{\text {eff }}(\phi)$ with an effective cosmological constant $\Lambda \simeq 10^{-122} M_{p}^{2}$ and the Planck mass $M_{p} \simeq 10^{19} \mathrm{GeV}$. We suppose that the BD field $\phi$ would be an attractor in the asymptotic region with $\phi \sim M_{p}$. Note that $\left\langle\phi^{2}>\sim 1 / G\right.$ in the BD theory [7] with its Lagrangian density, $\phi^{2} R-\omega g^{\alpha \beta} \partial_{\alpha} \phi \partial_{\beta} \phi-V(\phi)$. Taking the heavy field mass $m_{h} \simeq 10^{-17} M_{p}$ and $M_{d} \simeq 10^{-x} M_{p}$ in Eqs. (13) and (16), we have the useful solutions in (possibly smallest) integers $l$ and $k$ :

Interesting cases, [ $l=3, k=3, x=5$ ] and [ $l=3, k=6, x=2$ ], of natural numbers $k, l$, and $x$ can be related with effective theories, which are derived from a grand unified theory defined at energy higher than $M_{d} \simeq 10^{14} \mathrm{GeV}$ or $M_{d} \simeq 10{ }^{17} \mathrm{GeV}$, respectively. In these cases,

$$
\mathcal{V}^{e f f}(\phi) \propto m_{h}^{6} / M_{d}^{2} \cdot\left(M_{d} / \phi\right)^{2 k} .
$$

Other cases of [ $l=3$ ] and [ $k=$ any integer, $x=0$ ] are obtained with a little different values of $m_{h} \sim$ $10^{-20} M_{p}$ and $\Lambda \sim 10^{-120} M_{p}^{2}$, compared to the former cases. In the latter cases, the non-renormalizable 
interaction term of the BD and the heavy field in Eq. (10) is induced from a high-energy theory which resides at energy near $M_{p}$. The effective potential is $\mathcal{V}^{e f f}(\phi) \propto m_{h}^{6} / M_{p}^{2} \cdot\left(M_{p} / \phi\right)^{2 k}$.

We can restate that, in the low-energy effective theory of BD gravity coupled with a heavy field whose non-renormalizable interaction terms are derived from a grand unified theory, we get a petite cosmological constant $\Lambda$ from the effective potential $\mathcal{V}^{e f f}$ in Eq. (16) (with suitable $m_{h}$ and $M_{d}$ ). The cosmological constant problem thus can be alleviated in this kind of quintessence model with the inverse power law, effective potential of the BD field in the late-time universe, as given in Eqs. (13) and (17).

\section{Acknowledgements}

We would like to thank Profs. Y. M. Cho, R. Ruffini, S. W. Kim, S. P. Kim, H. Y. Lee, and G. Kang, who invite us. We thank Profs. C. Lee, H. Min, and P. Y. Pac who helped us learn field theories. We also thank Profs. M-S Kim and H-Y Park who were our colleagues in Soongsil University.

It was supported by the Basic Science Research Program through the National Research Foundation of Korea (NRF) funded by Ministry of Education, Science and Technology (NRF2017R1D1A1B06032249).

\section{References}

[1] Riess A. G. et al., Astron. J. 116, 1009 (1998); Perlmutter S. J. et al, Astrophys. J. 517, 565 (1999); Perlmutter S. J., Turner M. S., and White M. J., Phys. Rev. Lett. 83, 670 (1999); Riess A. G. et al., Astrophys. J. 659, 98 (2007); Spergel D. N. et al. [WMAP Collaboration], Astrophys. J. Suppl. 170, 377 (2007).

[2] Mannheim P. D., Astrophys. J. 479, 659 (1997); Lee T. H. and Lee B. J., Phys. Rev. D 69, 127502 (2004).

[3] Ratra B. and Peebles P. J. E., Phys. Rev. D 37, 3406 (1988); Frieman J. and Waga I., Phys. Rev. D 57, 4642 (1998); Steinhardt P. J., Wang L., and Zlatev I., Phys. Rev. D 59, 123504 (1999).

[4] Caldwell R. R., Phys. Lett. B 545, 23 (2002).

[5] Lee C., Lee T. H., and Min H., Phys. Rev. D 39, 1681 (1989); 1701 (1989).

[6] Damdinsuren B., Sim J., and Lee T. H., Class. Quant. Grav. 34, 175012 (2017).

[7] Brans C. and Dicke R., Phys. Rev. 124,925 (1961). 